\title{
Comparison to a Prior ECG
}

National Cancer Institute

\section{Source}

National Cancer Institute. Comparison to a Prior ECG. NCI Thesaurus. Code C117761.

A comparative interpretation of an ECG relative to a previous (comparator) ECG. The definition of the comparator ECG may be specified elsewhere. Common comparator result values include improved, no change, deteriorated. (CDISC) 